\title{
An Efficient Detection of Kidney Stone Based on HDVS Deep Learning Approach
}

\author{
Krishnamoorthy Somasundaram ${ }^{1, *}$, Suresh Manimekalai ${ }^{2}$, Paulraj Sivakumar ${ }^{3}$ \\ \{sks.it@psgtech.ac.in ${ }^{1}$, manimekalaisuresh06@gmail.com², psk.it@psgtech.ac.in $\left.{ }^{3}\right\}$ \\ Assistant Professor (Selection Grade), Department of Information Technology, PSG College of \\ Technology, Coimbatore, Tamil Nadu, India ${ }^{1}$, Assistant Professor, PSNA College of Engineering and \\ Technology, Dindigul, Tamil Nadu, India ${ }^{2}$, Assistant Professor (Senior Grade), Department of \\ Information Technology, PSG College of Technology, Coimbatore, Tamil Nadu, India ${ }^{3}$
}

\begin{abstract}
The Kidney stones (KDS) are the most normal issues in the universe that humans are affected with a high pain and also need an emergency diagnosis. To detect the KDS in an early stage for diagnosis, there are several imaging techniques are provided. For a perfect imaging, the Computer-aided diagnosis (CAD) methods are performed as auxiliary tools to for the support during KDS diagnosis. The computed tomography (CT) images are recently used for an exact diagnosis of KDS. But in the CT images, the prediction accuracy is less with the usage of traditional techniques. Therefore, the deep learning (DL) methods are well versed on handling such images with a greater accuracy. In this paper, the combination of DL methods to perform a both feature extraction and the classification for KDS detection is presented. The feature is extracted with the DL method of Visual Geometry Group network 19 (VGN-19) which is based on convolutional neural network $(\mathrm{CNN})$. Then the classification is done by the Binary Support Vector Machine classifier (SVM) to build a binary model. Thus the proposed model is named as a Hybrid Deep VGN-19 and Binary SVM (HDVS) which is an efficient in the disease prediction with a performance metric of of Recall, precision, accuracy, specificity and F1 score respectively. These performances of proposed HDVS method are compared with the prior advance methods in DL approaches such as Alexnet, GoogleNet, ResNet and SqueezeNet.
\end{abstract}

Keywords: kidney stone, Deep learning, Performance metrics, Binary SVM, VGN-19.

\section{Introduction}

In recent days, KDS is the normal issues that occurred in a human health in every country with a little variation in numbers. This KDS is a disease that may leads to minimise the function of kidney and cause a heavy pain in the body. This KDS affected the kidney to trouble its process and also reduce the human's life durations. This KDS initially affects the urinary system and finally leads to death by a kidney failure. According to the report, the KDS is occurred in $11 \%$ of men and $9 \%$ of women in recent statistics [1]. Similiar the other disease like blood pressure, obesity and diabetes are also troubles the KDS.

The only solution is an early diagnosis can save the life of human and can prevent from the kidney failure. Several imaging methods are used for early detection the KDS such as computed tomography (CT), magnetic resonance imaging (MRI) and X-ray. These imaging 
techniques are used for robust and accurate KDS prediction [2]. To improve the better performance of the system and also early detection and diagnosis, the DL approaches are used.

The DL approaches are the most significant for the prediction which is highly accurate and sensitive in learning and training the data. The DL is very useful for the medical data prediction that are applied by the many researchers and proved it. some of the DL applied medical fields are cardiac arrhythmia, brain tumor, Covid-19, , prostate cancer, KDS, skin cancer and breast cancer [3]. Therefore the most advance techniques of DL like Convolutional Neural Network (CNN), Recurrent NN (RNN), UNet, Alexnet, GoogleNet, ResNet and SqueezeNet are also applied to various medical fields and performed well in the diagnosis.

In this work, the proposed HDVS model is constructed for the prediction of KDS disease for early detection and diagnosis. This model utilized a CT images for the prediction accuracy and robust with a minimum error using the proposed model. The proposed model is to perform a both feature extraction and classification for the KDS prediction. Therefore the VGN-19 is applied to extract the features and Binary SVM is used for the classification. Thus the performance is evaluated with the prior model comparison with a higher accuracy and low computational cost.

This work is organised as follow: the section 2 presented the related works that are performed for KDS and in the section 3, the preliminaries are presented with the basics of VGN-19 and Binary SVM. The section 4 discussed the proposed methodology with a full flow diagram and the section 5 described the result with the comparisons. The section 6 is ended the work with conclusion.

\section{Related Works}

This section discussed that the related work towards the KDS detection from a several researchers are presented in it. Akshaya, $\mathrm{M}$ et al [4] have proposed a multi layer perceptron based neural network for stone detection in kidney images. The features are extracted using fuzzy c means algorithm. Then feed forward network is used for classification.

Thein, $\mathrm{N}$ et al [5] have proposed a thresholding based algorithm for kidney image processing. The threshold levels based on intensity, size and location to clear the un wanted portions. Results show that the proposed thresholding approach achieves $89.9 \%$ specificity when compared to other methods. Soni, A et al [6] have proposed ensemble learning approach for kidney stone detection. The extracted features classified using support vector machine for accurate localization. Implementation results on kidney CT image data set shows that the proposed approach increases the accuracy level of $98.7 \%$ than other methods. Hafizah, W. M et al [7] have proposed an accurate kidney stone detection method using five level histogram features. The gray level co-occurrence matrix calculated for segmenting the portions accurately. For pre-processing, contour detection based technique has been used.

Myint, L. Y et al [8] have proposed a hypodense and isodense region based region processing algorithms for kidney stone segmentation and classification. For each region, intensity thresholding applied to segment useful portions. Marsousi, $\mathrm{M}$ et al [9] have presented structure trained kidney stone classification model for ultra sound kidney images. Further, the probabilistic kidney shape model is generated by using mean and variance features. When compared to other methods, the proposed method shows $9369 \%$ of accuracy for all data set images. 
Harsh Dave et al [10] have proposed a correlation and entropy based kidney stone segmentation for ultrasound images. For segmentation, second angular moment has been used. For classification, KNN classifier is used. The proposed model shows superior performance all parameters when compared with traditional approaches. Priyanka et al [11] have developed a principal component analysis combined segmentation model for effective stone segmentation. The gray level feature of entropy, energy and moment used for training and principal component analysis used for classification. The proposed hybrid model achieves higher accuracy than SVM and KNN models.

Viswanath, $\mathrm{K}$ et al [12] have analysed an area and power of kidney image processing algorithms for chip level implementation. To extract a feature, level set segmentation has been used. For classification, artificial neural network has been used. Implementation results on FPGA shows that the proposed models occupy less area and time for pixel processing. Kazemi, Y et al [13] have developed an ensemble learning model kidney stone detection. The hyper parameters of the model is tuned by using optimization algorithms. The execution time is complex when increasing number of layers in an ensemble model. Guo, Z. et al [14] have developed a deep learning model for xray, MRI and CT medical image segmentation. It combines fusion techniques to extract a relevant feature. The proposed model shows higher dice coefficient similarity values when compared with ground truth images. Gridach, $\mathrm{M}$ et al 2021 have proposed a deep net model for medical image segmentation. The layer level modification is performed to increase a feature extraction capability of the network.

\section{Preliminaries}

In this section, the basic idea of VGN-19 and the binary SVM is described in brief.

$V G N$

The VGN is based on the transfer learning DL method which is developed at 2014. This model is so easy and iterative architecture. This model has satisfied in ImageNet Large-Scale Visual Recognition Challenge (ILSVRC) 2014 of error rate as $7.32 \%$. This model is similar to the AlexNet and also used an additional convolution layers (CVL). It is invented by the Oxford University's Visual Geometry Group (VGG). It has a 16-19 CVL which is a dense network of deep CNN by loading $2 \times 2$ high pool layer and $3 \times 3$ small CVL cores frequently.

\section{Binary SVM}

SVM is the important learned methods that are utilized for complex issues which is from the statistical learning theory. It has a data point with a high dimension that provides a various linear classes to build an optimal decision based on hyperplane. This hyperplane used to differentiate the data point in this space. By performing kernel calculations (KCs), the quadratic programming can be rectified. Some of the KCs are polynomials, linear and radial basis function is accessed with SVM. Compared with an other methods, the SVM is simple to construct and efficient in decision making.

For instance, the $\mathrm{N}$ data points are provide for training dataset such as $\mathrm{A}=\{\mathrm{a} 1, \mathrm{a} 2, \ldots \mathrm{aN}$ \}. Every pattern ai $\in \mathrm{Rn}$ which also have a 'd' features and has a two classes yi $\in\{ \pm 1\}$. When the patterns are linearly separable for trainning, a hyperplane can be splited up the feature space data which is given in the below expression.

$$
\mathrm{f}^{\prime}(\mathrm{a})=\mathrm{W}^{\mathrm{T} \prime} \mathrm{a}+\mathrm{b}^{\prime}=0
$$


Where w' indicates an adjustable weight vector and b' indicates a bias

\section{Proposed Methodology}

This section provides an overall proposed HDVS architecture that includes an entire process for the KDS prediction which is given in the figure 1 . The proposed architecture consists of input of dataset, pre-processing, feature extraction, classification and prediction respectively. The every blocks of the proposed methodology is explained in the following.

Dataset description

The KDS datasets are gathered from the websites of https:/github.com/yildirimozal/Kidney_stone_detection that has an overall 433 reports of people. It contains a $278 \mathrm{KDS}$ positive and 165 negative (normal) based on the CT images. It has been collected a various patients CT images upto 790 KDS diagnosed and 1009 normal cases in it.

\section{Pre-processing}

These data is splatted for both testing and training where the $70 \%$ of data used for training and $30 \%$ data used for testing. The both the evaluation are entirely various from each other. This data is carried for the extraction and classification to predict the KDS with accuracy. Therefore, the training data is processed a VGN-19 feature extraction and then performed a Binary SVM classification to provide an exact detection.

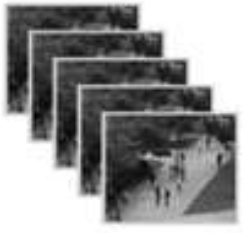

Normal and Abnormal Frames

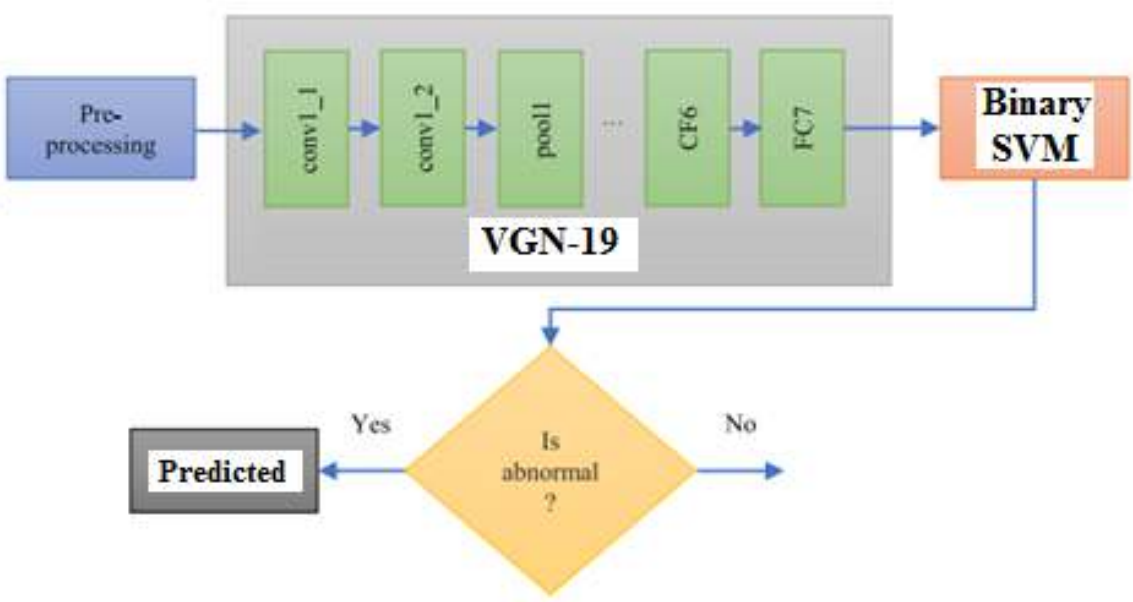

Figure 1 The Proposed Hybrid architecture 


\section{Proposed HDVS Technique}

After performing a pre-processing, the training data are employed to proposed HDVS block. This block contains a VGN-19 initially to perform a feature extraction. This model fit a small sized data and omits a bigger data which reduces the trained data volume. The VGN-19 is used to form a pre-trained model to achieve a low computation and low cost. Then the data is sent to perform a Binary SVM classifier which is used to classify the data as normal and abnormal. This trained data are carried out with an accurate prediction and achieved a higher system performance. Thus the proposed is explained in the following.

\section{i) VGN-19 FEATURE EXTRACTION}

The VGN-19 method [16] is used to extract the KDS data features in this system. It provides a 19 layered depth and trained by an ImageNet dataset. It has an input data of $224 \times 224$ RGB images. This model has a 5blocks of CVL and three fully connected layers. every CVL has a $3 \times 3$ kernel to enhance the system accuracy. The stride 1 provides an one padding with an activation map that has an equal spacing. It has a 5 max-pooling layer for down sampling which perform a stride 2 with $2 \times 2$ windows. It contains zero padding by splitting a former layer of each spatial dimension. Every CVL has Relu which is an activation function than connected with fully connected layers. Then the features of KDS are extracted and an activation map is generated.

\section{ii) BINARY SVM CLASSIFIER}

The extracted features are moved to perform a classifier using a binary SVM model [17]. The Binary SVM with linear kernel are used to perform the classification which capable of solving a complex issues. it can perform a larger dataset for the prediction but this model choose only two parameter for SVM implementations such as kernel and upperbound parameters. On performing a structural risk minimization (SRM) principle, this model is generalized. This learning method has an output labels based on hyperplane separation. When the two input space classes are separated linearly, then the hyperplane is expressed as:

$\{\mathrm{xi}, \mathrm{yi}\}_{\mathrm{i}=1}^{\mathrm{n}}, \mathrm{yi} \in\{ \pm 1\}(2)$

where yi $\in\{ \pm 1\}$ has a input vector $\mathrm{x}$ as classification "label".

Then the Binary SVM is expressed in the following.

$\mathrm{y}^{\prime}(\mathrm{x})=\operatorname{sig}\left[\sum_{\mathrm{i}=1}^{\mathrm{n}} \propto\right.$ i. $\mathrm{yi} . \mathrm{k}(\mathrm{x}, \mathrm{xi})+\mathrm{b}^{\prime}$

where $\alpha \mathrm{i}$ indicates a multiplier of Lagrange and yi indicates a training pattern xi target value. the $\mathrm{k}(\mathrm{x}, \mathrm{xi})$ represents the kernel function and $\mathrm{b}$ ' indicates the bias.

Prediction

Finally the classification result is used to split the KDS CT images and normal images with a higher accuracy.

\section{Result And Discussion}

This section presented the experimental results for the proposed HDVS methods. The datset is obtained and splited with a testing and testing data to perform a KDS detection. These performances of proposed HDVS method are compared with the prior advance methods in DL 
approaches such as Alexnet, GoogleNet, ResNet and SqueezeNet. The performance metric of HFDVS methodology is discussed interms of Recall (Rec'), precision (Prec'), accuracy (A'), specificity (Spc') and F1 score (F1s') respectively. The expressions to evaluate these metrics are given below.

$$
\begin{aligned}
& \operatorname{Prec}^{\prime}=\frac{\mathrm{T}_{\mathrm{P}}}{\mathrm{T}_{\mathrm{P}}+\mathrm{F}_{\mathrm{P}}}=1-\mathrm{P}_{\mathrm{f}}^{\prime} \\
& \operatorname{Rec}^{\prime}=\frac{\mathrm{T}_{\mathrm{P}}}{\mathrm{T}_{\mathrm{P}_{\mathrm{P}}+\mathrm{F}_{\mathrm{N}}}} \\
& \mathbf{S p c}^{\prime}=\frac{\mathrm{T}_{\mathrm{N}}}{\mathrm{T}_{\mathrm{P}}+\mathrm{T}_{\mathrm{N}}} \\
& \mathbf{A}^{\prime}=\frac{\mathrm{T}_{\mathbf{P}}+\mathrm{T}_{\mathbf{N}}}{\mathrm{T}_{\mathbf{P}}+\mathbf{T}_{\mathbf{N}}+\mathbf{F}_{\mathbf{P}}+\mathbf{F}_{\mathbf{N}}}
\end{aligned}
$$

Where $\mathbf{P}_{\mathbf{f}}$ indicates the probability of false detection, $\mathbf{T}_{\mathbf{P}}$ represents the True-Positive, $\mathbf{T}_{\mathbf{N}}$ represents the True-Negative, $\mathbf{F}_{\mathbf{P}}$ represents the False-Positive and $\mathbf{F}_{\mathbf{N}}$ indicates the FalseNegative.

The F1s' is the recall's average harmonic and precision value ratios which has a value of

\begin{tabular}{|c|c|c|c|c|c|}
\hline Method & Accuracy & $\mathrm{Spc}^{\prime}$ & Prec $^{\prime}$ & $\operatorname{Rec}^{\prime}$ & F1s' \\
\hline AlexNet & 99.19 & 98.28 & 98.07 & 97.32 & 0.932 \\
\hline GoogleNet & 71.65 & 67.23 & 54.03 & 72.78 & 0.965 \\
\hline ResNet & 96.86 & 98.74 & 97.45 & 98.64 & 0.912 \\
\hline SqueezeNet & 98.89 & 97.63 & 98.09 & 98.89 & 0.901 \\
\hline Proposed HDVS & 99.89 & 99.87 & 99.76 & 99.51 & 0.990 \\
\hline
\end{tabular}
0 as worst as 0 and 1 as best.

$$
\mathrm{F} 1 \mathrm{~s}^{\prime}=\frac{2 \text { Prec } \prime \times \text { Rec } \prime}{\text { Prec } \prime+\text { Rec } \prime}
$$

Table 1: Comparison of DL methods

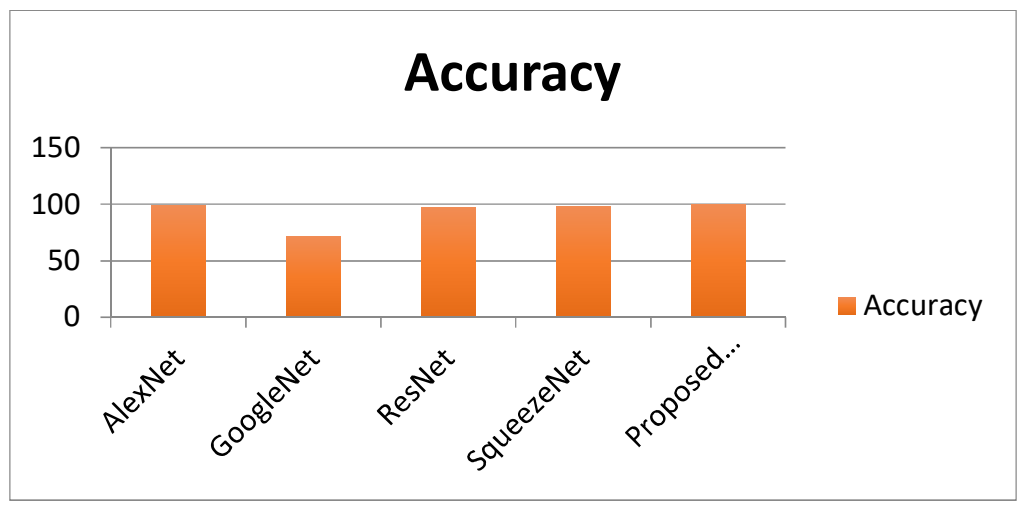

Figure 2 Accuracy performance chart 


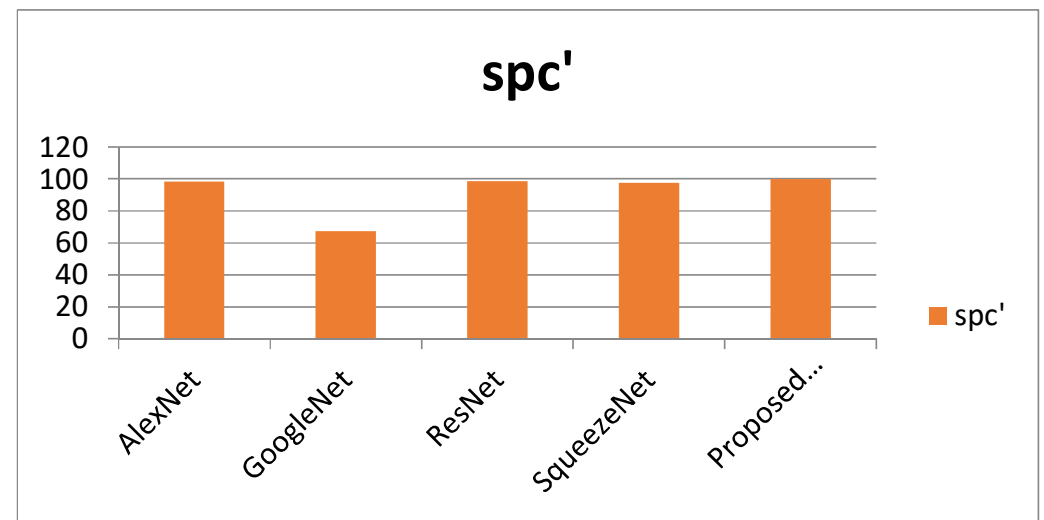

Figure 3 specificity performance chart

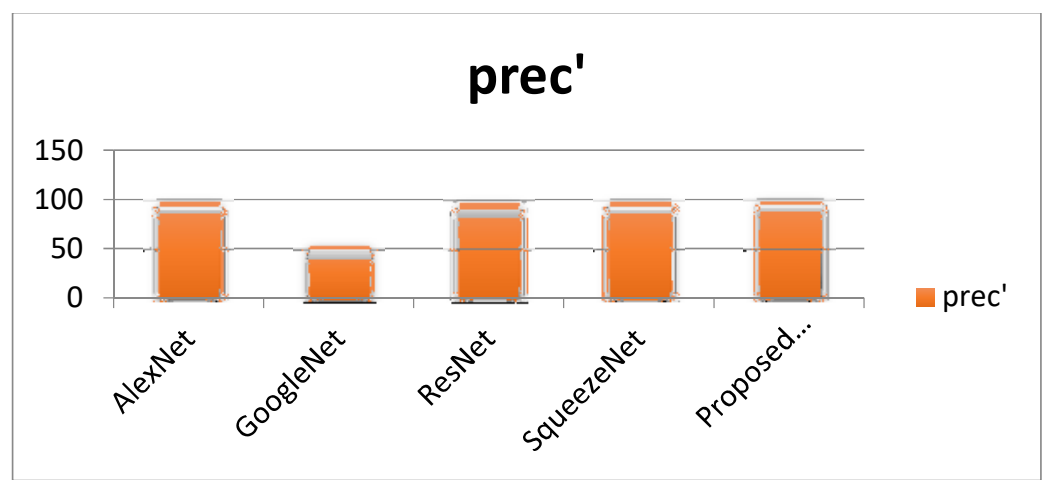

Figure 4 precision performance chart

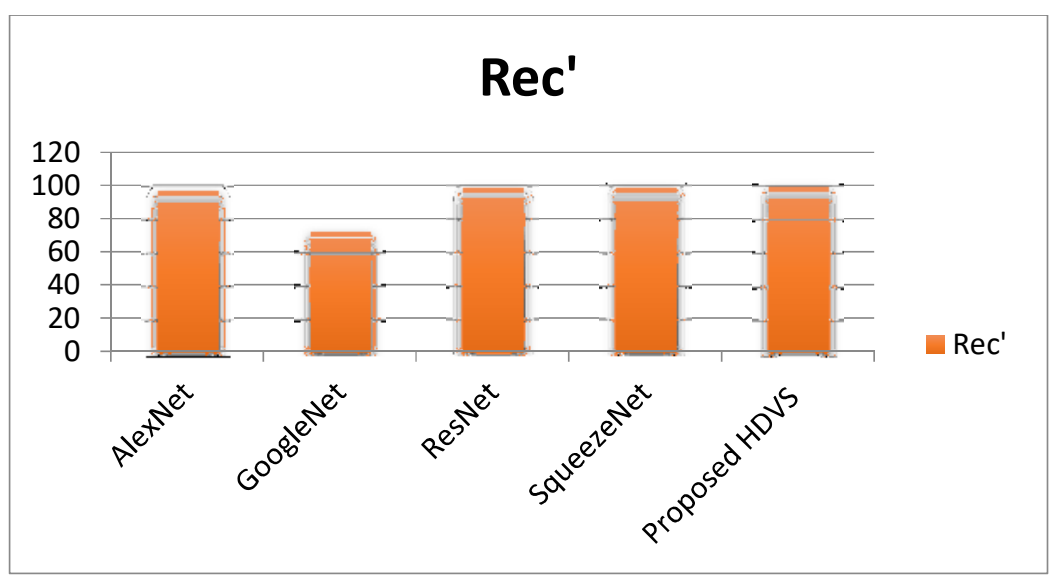

Figure 5 Recall performance chart 


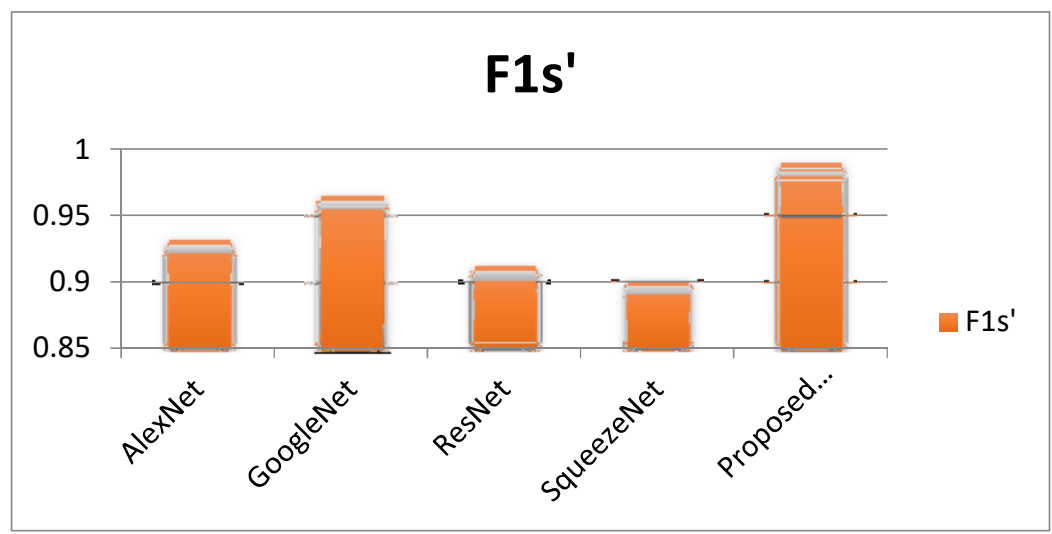

Figure 6 F1s' performance chart

Based on the table 1 and figure 2 to 6 , the performance of proposed HDVS and the prior DL methods of Alexnet, GoogleNet, ResNet and SqueezeNet are evaluated. In this experiment, the performance metric of Recall (Rec'), precision (Prec'), accuracy (A'), specificity (Spc') and F1 score (F1s') are evaluated. The result showed that the proposed HDVS made a good and accurate performance in all the metrics which can be efficient in detection.

\section{Conclusion}

This work presented the KDS detection based on DL method of HDVS technique that combined both the VGN-19 and Binary SVM respectively. By performing a HDVS methodology, the KDS obtained is predicted with a higher accuracy in a n early stage. This method can be compared with a prior model of DL approaches Alexnet, GoogleNet, ResNet and SqueezeNet. Based on the result, the proposed HDVS can be performed well in all the part of prediction in terms of Recall, precision, accuracy, specificity and F1 score. In future, the proposed model of HDVS can be applied to all the medical data to provide a better diagnosis.

\section{References}

[1] M. Noll, X. Li, and S. Wesarg, "Automated kidney detection and segmentation in 3D ultrasound," in Proc. Workshop Clin. Image-Based Procedures, Sep. 2014, pp. 83-90.

[2] V. F. van Ravesteijn, C. van Wijk, F. M. Vos, R. Truyen, J. F. Peters, J. Stoker, and L. J. van Vliet, "Computer-aided detection of polyps in CT colonography using logistic regression," IEEE Trans. Med. Imag., vol. 9, no. 7, pp. 120-131, Jan. 2019

[3] S. Zheng, J. Guo, X. Cui, R. N. J. Veldhuis, M. Oudkerk, and P. M. A. van Ooijen, "Automatic pulmonary nodule detection in $\mathrm{CT}$ scans using convolutional neural networks based on maximum intensity projection,"' IEEE Trans. Med. Imag., to be published.

[4] Akshaya, M., Nithushaa, R., Raja, N. S. M., \& Padmapriya, S. (2020). Kidney Stone Detection Using Neural Networks. 2020 International Conference on System, Computation, Automation and Networking (ICSCAN). 
[5] Thein, N., Nugroho, H. A., Adji, T. B., \& Hamamoto, K. (2018). An image preprocessing method for kidney stone segmentation in CT scan images. 2018 International Conference on Computer Engineering, Network and Intelligent Multimedia (CENIM).

[6] Soni, A., \& Rai, A. (2020). Kidney Stone Recognition and Extraction using Directional Emboss \& SVM from Computed Tomography Images. 2020 Third International Conference on Multimedia Processing, Communication \& Information Technology (MPCIT).

[7] Hafizah, W. M., Supriyanto, E., \& Yunus, J. (2012). Feature Extraction of Kidney Ultrasound Images Based on Intensity Histogram and Gray Level Co-occurrence Matrix. 2012 Sixth Asia Modelling Symposium.

[8] Myint, L. Y., Maung, S. S., \& Zar, K. T. (2020). Removal of Unwanted Object in 3D CT Kidney Stone Images and 3D Visualization. 2020 24th International Computer Science and Engineering Conference (ICSEC).

[9] Marsousi, M., Plataniotis, K. N., \& Stergiopoulos, S. (2014). Shape-based kidney detection and segmentation in three-dimensional abdominal ultrasound images. 2014 36th Annual International Conference of the IEEE Engineering in Medicine and Biology Society.

[10] Harsh Dave; Vaishnavi Patel; Jay N Mehta ,Regional Kidney Stone Detection and Classification In Ultrasound Images, 2021 Third International Conference on Inventive Research in Computing Applications (ICIRCA)

[11] Priyanka, \& Kumar, D. (2020). Feature Extraction and Selection of kidney Ultrasound Images Using GLCM and PCA. Procedia Computer Science, 167, 1722-1731.

[12] Viswanath, K., Gunasundari, R., \& Hussan, S. A. (2015). VLSI Implementation and Analysis of Kidney Stone Detection by Level Set Segmentation and ANN Classification. Procedia Computer Science, 48, 612-622.

[13] Kazemi, Y., \& Mirroshandel, S. A. (2018). A novel method for predicting kidney stone type using ensemble learning. Artificial Intelligence in Medicine, 84, 117-126.

[14] D. S. Vijayan, A. Leema Rose, S. Arvindan, J. Revathy, C. Amuthadevi, "Automation systems in smart buildings: a review", Journal of Ambient Intelligence and Humanized Computing https://doi.org/10.1007/s12652-020-02666-9

[15] Gridach, M., \& Voiculescu, I. (2021). Dopnet: Densely Oriented Pooling Network For Medical Image Segmentation. 2021 IEEE 18th International Symposium on Biomedical Imaging (ISBI).

[16] J. Jaworek-Korjakowska, P. Kleczek and M. Gorgon, "Melanoma Thickness Prediction Based on Convolutional Neural Network With VGG-19 Model Transfer Learning," 2019 IEEE/CVF Conference on Computer Vision and Pattern Recognition Workshops (CVPRW), 2019, pp. 27482756, doi: 10.1109/CVPRW.2019.00333.

[17] M. Tholkapiyan, A.Mohan, Vijayan.D.S , "A survey of recent studieson chlorophyll variation in Indian coastal waters", IOP Conf. Series: Materials Science and Engineering 993 (2020) 012041, doi:10.1088/1757-899X/993/1/012041.

[18] D.Suresh, D.Ramesh, S Satheesbabu, S,K.Somasundaram, "Removing Speckle Noise in Synthetic Aperture Radar Images using Combined Intensity Coherence Vector \&Hybrid Filtering", $\begin{array}{lllll}\text { PSYCHOLOGY AND EDUCATION } & \text { (2021) 58(1): }\end{array}$ https://doi.org/10.17762/pae.v58i1.1497

[19] Somasundaram, K., Sivakumar, P., Suresh, D. "Classification of Diabetic Retinopathy Disease with Transfer Learning using Deep Convolutional Neural Networks"(2021) Advances in Electrical and Computer Engineering, 21 (3), pp. 49-56. DOI: 10.4316/AECE.2021.03006. 